American Chemical Society, J. Phys. Chem. C., jp0710772w, Supporting Information Page S1

\title{
Monodisperse Particles of Bifunctional Periodic Mesoporous Organosilica
}

\section{Eun-Bum Cho, ${ }^{\dagger}$ Dukjoon Kim, ${ }^{\dagger *}$ Mietek Jaroniec $^{\ddagger *}$}

${ }^{\dagger}$ Polymer Technology Institute, Department of Chemical Engineering, Sungkyunkwan University, Suwon, Gyeonggi-do 440-746, Korea

${ }^{\ddagger}$ Department of Chemistry, Kent State University, Kent, Ohio, 44240 USA
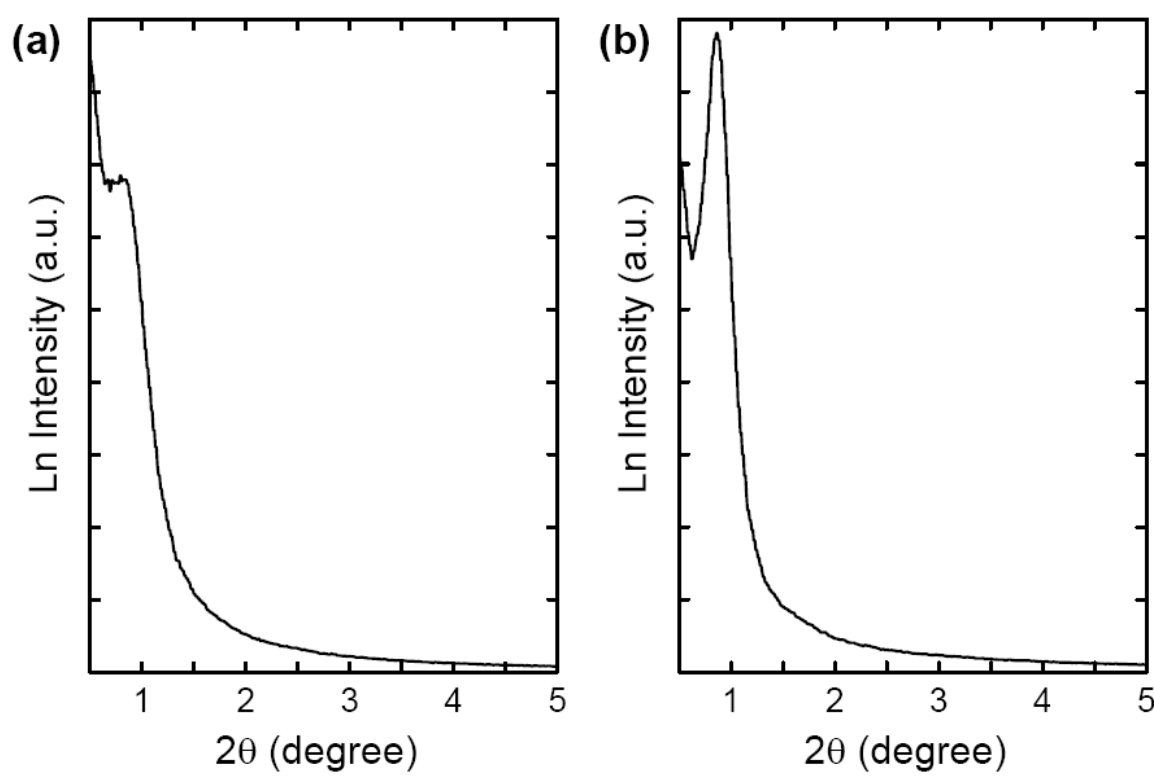

Figure S1 SAXS patterns for ethane-benzene-PMOs prepared using P123 triblock copolymer, BTEE, and BTEB. The relative molar ratios of BTEE and BTEB are (a) 90:10 and (b) 80:20, respectively. 
American Chemical Society, J. Phys. Chem. C., jp0710772w, Supporting Information Page S2

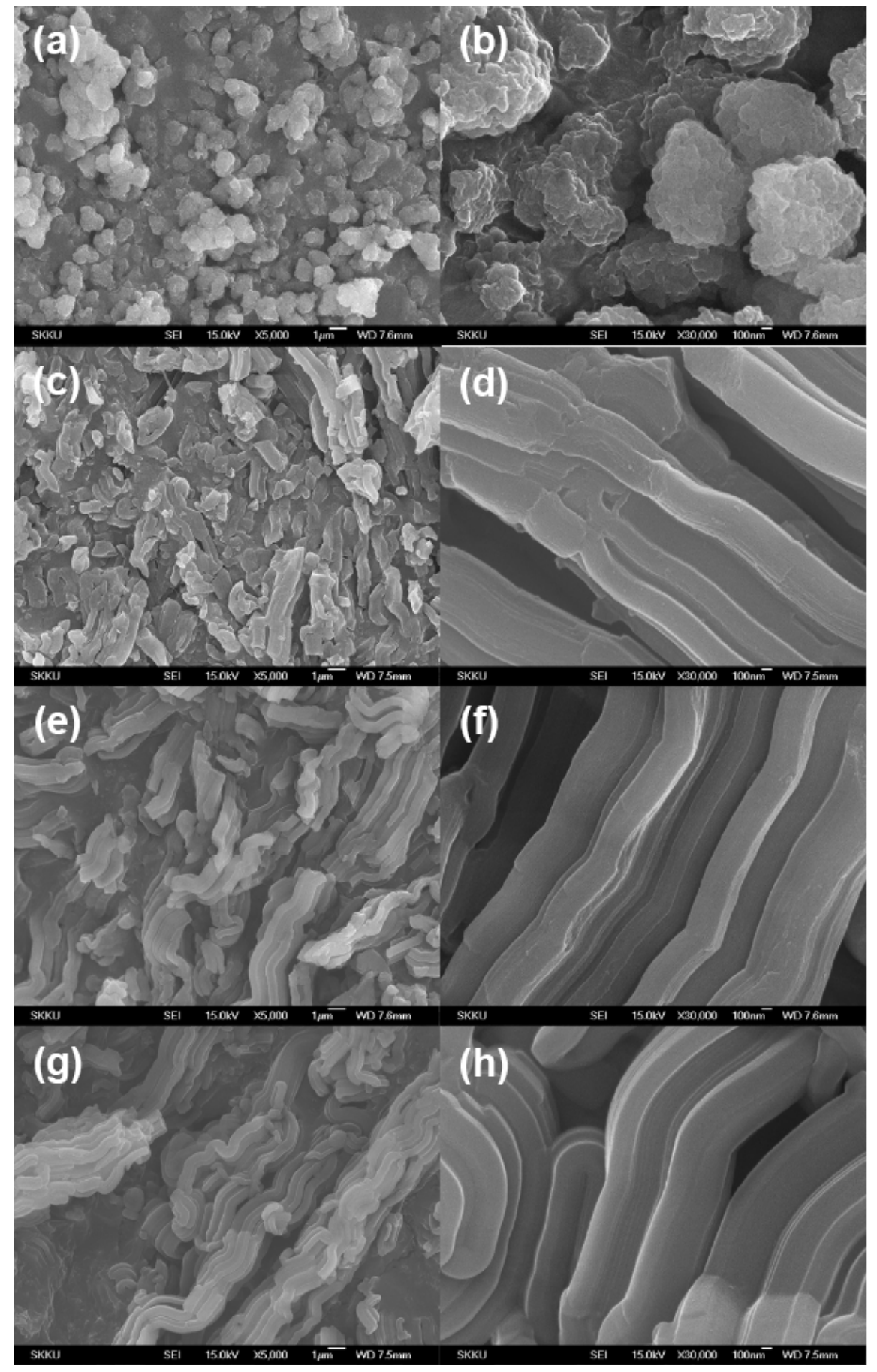

Figure S2. SEM images for ethane-benzene-PMOs obtained for the following BTEE:BTEB molar ratios: 100:0 (a,b), 40:60 (c,d), 20:80 (e,f) and 0:100 (g,h). 